\title{
The Many Vortexes of NGC 5236 Nucleus in The Central $80 \times 200$ Parsecs
}

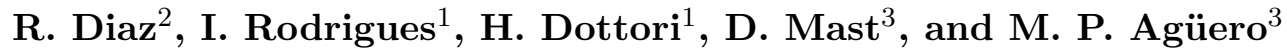 \\ ${ }^{1}$ Instituto de Física, UFRGS,cp: 15051, cep: 91501-970, Porto Alegre, Brazil \\ email: dottori@if.ufrgs.br \\ ${ }^{2}$ Gemini Observatory, Southern Operations Center, Chile. \\ CASLEO, CONICET, Argentina. \\ e-mail:rdiaz@gemini.edu \\ ${ }^{3}$ Observatório Astronómico de Córdoba, Laprida 854, Córdoba, Argentina
}

\begin{abstract}
GEMINI-S+CIRPASS configuration has been used to obtain 490 high quality spectra, centred in 1.3 microns of the NW-SE oriented, central $80 \times 200$ pc of NGC 5236 (M83), with spectral resolution of 3200 . We determine the kinematics of this region with 0.36 arcsec sampling, sub-arcsec resolution. Disk-like motions are detected in $\mathrm{Pa} \beta$ at parsec scales around: a) the optical nucleus $(\mathrm{ON}), \mathrm{b})$ the dynamical centre of the $\mathrm{CO}$ velocity map (Sakamoto et al. 2004) coincident with the K-band center (hereafter KC; Thatte et al. 2000), located 50 pc to the $\mathrm{W}$ of the optical nucleus, and c) the hidden condensation (hereafter HN; Mast et al. 2006), now more precisely located at $120 \mathrm{pc}$ to the N-NW of the optical nucleus. The disk around ON has a radius of $10-15 \mathrm{pc}$ and those around $\mathrm{KC}$ and $\mathrm{HN}$ can be traced approximately up to $40-50$ pc from their kinematical centres. Rotation curve fittings using Satoh like spheroids give masses of $2-4 \times 10^{6} \mathrm{M}_{\odot}, 10-15 \times 10^{6} \mathrm{M}_{\odot}$ and $15-20 \times 10^{6} \mathrm{M}_{\odot}$ respectively. N-body simulations using Gadget2 (Springel, 2005) show that ON, KC and HN will merge in 20-50 Myrs. A question that arise immediately is if this degree of dynamical activity is peculiar of M83 or it is a common behaviour, seen in this galaxy in all its dramaticity due to its small distance from us.
\end{abstract}
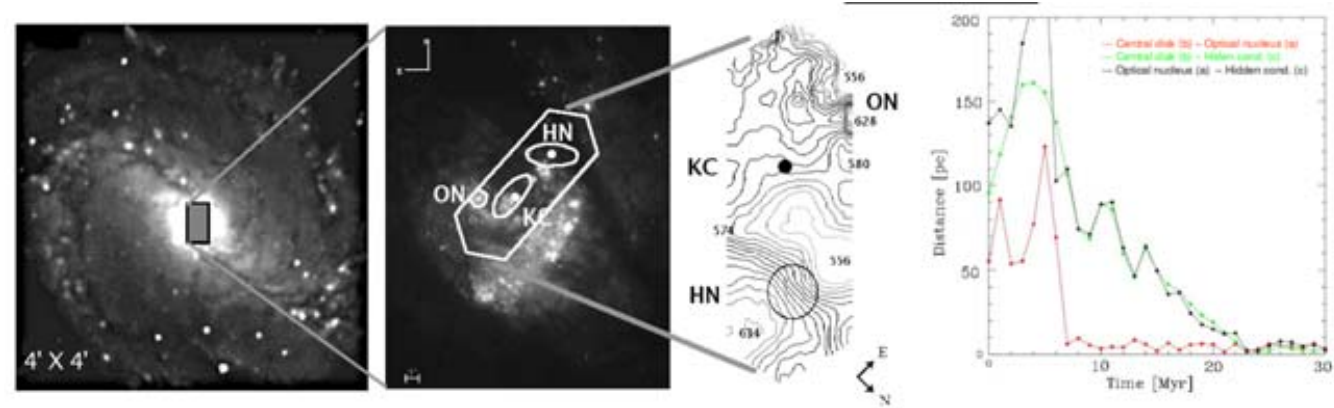

Figure 1. From left to right: a) Large scale image of M83; b) Hst image of the central part outlining the CIRPASS field; c) $\mathrm{Pa} \beta$ isovelocity contours, showing the positions of $\mathrm{ON}, \mathrm{KC}$ and $\mathrm{HN}$; d) Time evolution of the distance $\mathrm{KC}-\mathrm{ON}, \mathrm{KC}-\mathrm{HN}$ and $\mathrm{ON}-\mathrm{HN}$, from one of our N-body simulations: all structures will merge in $23 \mathrm{Myr}$.

\section{References}

Diaz, R. J., Dottori, H., Mediavilla, E., Aguero, M., \& Mast, D. 2006, New Astron. Rev. 49, 547. Mast, D., Diaz, R., \& Aguero, M.P. 2006, AJ 131, 1394.

Sakamoto,K., Matsushita, S., Peck, A.B., Wiedner, M., \& Iono, D. 2004, ApJ (letters),616, 59. Springel, V. 2005, MNRAS 364, 1105.

Thatte, N., Tecza, M., \& Genzel, R. 2000, A\&A 364, 47. 